\title{
Cultural Beliefs, Social Support and Societal Ascribed Roles as Determinants of Patients' Perception of Illness Symptom
}

\author{
Felicia O. Owadara \\ Department of Social Work, Faculty of Education, University of Ibadan, Nigeria \\ *Corresponding Author: felicia.owadara@yahoo.com
}

\begin{abstract}
Given the roles of Patients' perception of illness symptom in recovery and response to treatment, this paper examines the influence of cultural beliefs, social support and roles ascribed to the patient by the society on their perception of illness symptom with the view to enhance it and obtain the associated advantages. The study was targeted at all the in-patients and out-patients of the forty-six state-owned hospitals of Oyo state in Nigeria, out of which nine hospitals were randomly selected. Fifty (50) in-patients and out-patients were selected by convenience sampling from each of the selected hospitals to participate in the study, making a total of 450 respondents. Only the responses from 370 respondents were usable as some did not complete the questionnaire properly or refused to give necessary and relevant information. The instrument for the study is a questionnaire titled questionnaire on patients perception of illness symptoms. Data collected were analyzed using descriptive statistics, t-test chi-square analysis, multiple regression analysis and Analysis of Variance (ANOVA). The results show that a considerable percentage of the patients were positive in their perception of their illnesses. These perceptions do not differ between male and female patients but are different on the basis of age. Societal ascribed roles seem to influence perception of illness than both cultural beliefs and social support either combined or individually.
\end{abstract}

Keywords Illness Symptom, Societal Ascribed Roles, Cultural Beliefs, Patients, Social Support

\section{Introduction}

Illness is commonly described as a condition of pronounced deviation from the normal healthy state (Dimatheo and Martin, 2002). The term is often used to mean disease but can also refer to a person's perception of their health, regardless of whether they in fact have a disease or not. A person without any disease may feel unhealthy and simply have the perception of having a disease. Another person may feel healthy with similar perceptions of perfectly good health.

In fact, illness is often not due to infection but a collection of evolved responses, illness behavior, by the body which aids the clearing of infection (Kelley, Bluthe, Dantzer, Zhou, Shen, Johnson \&Broussard, 2003). Such aspects of illness can include lethargy, depression, anorexia, sleepiness, hyperalgesia, and inability to concentrate (Johnson, 2002). However, Jennings (1986) argued that illnesses cannot be investigated by the methods of biomedicine because its study ultimately depends directly on phenomenological analysis of experienced suffering through individual self-reports and behavior and that its presence cannot be objectively established by physical signs.

When patients are diagnosed with an illness they generally develop an organized pattern of beliefs about their condition. These views are key determinants of behaviour directed at managing illness and these change in response to shifts in patients' perceptions and ideas about their illness. These illness perceptions or cognitive representations directly influence the individual's emotional response to the illness and their coping behaviour such as adherence to treatment. Despite their importance, patients' views of their illness or symptoms are rarely sought in medical interviews and patients tend not to bring up their illness beliefs with doctors.

According to Petrie and Weinman (2012), patients' models of their illness share a common structure made up of beliefs about the cause of an illness, the symptoms that are part of the condition, the consequences of the illness for the patient's life, how the illness is controlled or cured, and how long the illness will last. These beliefs are based either on their own medical knowledge and experience or on the experience of their friends or family members who have had similar symptoms or diagnoses (Petrie \& Weinmann, 2006). Also, patients with the same illness may have different perceptions of their condition and different emotional reactions to it (Broadbent, Petrie, Main \& Weinmann, 2006). 
Illness perceptions change rapidly in response to diagnostic results and have been associated with emotional distress, recovery, and disability, as well as with treatment-related behavior such as adherence. Interventions based around changing inaccurate or unhelpful perceptions of illness are an important emerging area of health psychology. These authors warned that patients' knowledge of medical concepts and the body is often rudimentary which can limit the accuracy and complexity of the models they build. However, it is important to note that a patient's perception of their illness can influence their coping ability, compliance with treatment and functional recovery. Psychological interventions to address negative beliefs may facilitate an earlier return to work (Giri, Poole, Nightingale and Robertson, 2009).

People routinely experience symptoms that may signal illness. Though symptoms are critical elements in seeking medical attention, however, the presence of symptoms is not always sufficient to prompt a visit to doctor (Cameron, Ellis, Petrie, Pennebaker, 1983). Some people seek help for symptom while some do not. When people seek and perceive symptoms of illness, they consult others such as friends, neighbour, family on whether or not to report the symptoms to the physicians. Some may be advised not do so until it is too complicated. Some denied the symptoms, pretend as if nothing happens to them, other result to self-medication. Some go to quacks doctors and may not be properly treated. There are quite a number of factors that influence when and how people look for help; symptoms themselves do not inevitably lead people to seek medical care but certain characteristics.

Illness symptoms are "differently labeled by individuals in dissimilar social situations". Certain biomedical etiologies maintain that symptoms are not part of the "patient's concept of his/her intact body" but are viewed as the manifestation of bodily malfunction. On the other hand, in non-traditional health care systems, symptoms are believed to be manifestations of the intrusion of the supernatural (Delea, 2006). Actually, most cultures support the belief that symptoms are the manifestation of illness, whether it is caused by a pathogen or a spirit invasion. Therefore, the symptom enables a person to report self-experiences of health on a day-to-day basis. She argued that, globally, people tend to use the symptom as a tool to communicate illness to the appropriate medical practitioner, regardless of the healing system or society.

Delea (2006) also gave an insight into the importance of illness symptoms. According to her, symptoms add clarity to the complex ideas of illness and healing in such a way that it is difficult to discuss either process without touching on these symbols. They are regarded as a vital part of the illness experience because they offer insight into the physiological and psychological aspects of the patient's body, such that, the symptom symbolizes the roots of a tree, anchoring a societal understanding of medical knowledge and healing etiologies.

Mechanic, (1978) listed characteristics that determine symptoms perceptions, these are listed below;

1. The visibility of the symptoms, that is, how readily apparent the symptoms are.

2. Perceived severity of the symptoms. Symptom perceived as severe will be more likely to prompt action than less severe ones.

3. Extent to which it interfere with personal life. The extent of the interference with personal life will prompt an individual to seek medical care as quickly as possible.

4. The frequency and persistency of the symptoms. Condition that people view as requiring care tends to be those that are severe and continuous therefore, intermittent symptoms are less likely than persistent symptoms that generate illness behaviour. According to Robert \& Pennebaker, (1995) severe symptoms prompt people to seek help from friends, family, physicians even mild symptoms prompt people to seek help when it persist.

Findings have shown that illness perception is associated with a number of outcomes in chronic illness, including self-management behaviours and quality of life. However, few interventions have been developed designed to change illness perceptions and improve illness outcome (Luszczynska, \& Schwarzer, 2005). Understanding cultural roles of the individual may help in our understanding of differences seeing in perception of symptoms by male and female. Another crucial issue was raised by Stroebe $\&$ Schut (2001), their findings showed that women are more conscious of their health and therefore participate in many more health promoting behaviours, such as preventive doctor visits, earlier detection of illness, and lower rates of high-risk behaviours. Johnson's report raised the awareness that women are becoming more susceptible to different health problems like coronary heart disease, because male have become the focus of research and women are less likely to be identified early as suffering from a heart attack and therefore receive the appropriate treatment at later stages of the disease which results in less effective endpoints (Johnson, 2002).

Again, recent research by Johnson have emphasized that there are evidences that social role may influence patients' perception of symptoms in term of decision in seeking medical care. Mechanic (1978) ascertained that in some cultures people are socialized to not reacting with strong reaction to illness. Whereas, in another culture, a strong reaction is expected, Jewish Americans were more likely to seek professional help, accept sick role and engage in preventive medical behaviour. While on the other hand, Mexican Americans tend to ignore some symptoms that professional feel are serious. Irish Americans tend to deny pains stoically. Other important factors that should be taken into consideration are; socio-economic factor, personal view of the illness, personal reluctance to seek medical care (Fieldman, 1966), age, and stress level.

It has been ascertained that women are more likely to use medical care system than men; the reason for this is somewhat complex. Pennibaker (1982) reported that women 
report symptom than men because of their sensitivity to internal body signal. It was asserted that, this was responsible for greater number of their reports. Malinowska-Sempruch, (2008) findings and that of (Verbrugie, 1989) also supported this fact, indicating growing number of concerns for this discrepancy.

Bendelow (2000) found out that women use more medical services and have higher rates of prescriptions compared with men. According to Bendelow (2002), gender differences in morbidity and mortality are biological aspects of illness risks, risks of illness acquired from gender roles, different health and illness reporting behaviours and differential diagnoses and treatments. In the epidemiological pattern of pain, women's pain is not always regarded as being as serious as men's. Fillingim (2000) stated that the female and male organisms differ in their response to pain. This is a fairly complex issue and there still is a lack of knowledge and understanding. As a result, different studies have presented various explanations of the gender differences, such as psychosocial explanations; "sex role expectancies (i.e. feminity vs. masculinity), cognitive/affective factors (e.g. anxiety, coping, and self-efficacy) and social learning".

Fillingim, (2000), concluded that gender-related factors are only some of many variables. Other factors such as age, disease and psychosocial status might be even more important. Gender differences in perceived chest pain and diagnosis have been found in several previous studies. Male patients more frequently than females were diagnosed with cardiac chest pain instead of non-cardiac chest pain (Keogh, Hamid, Hamid, \& Ellery, 2004; Omran \& Al-Hassan, 2006). Women with coronary heart disease symptoms presented more undifferentiated symptoms than men and women also had difficulty interpreting their symptoms, ending in delayed admission in hospitals (Lockyer, 2005; Omran \& Al-Hassan, 2006). When comparing men and women with cardiac diseases Nau, Ellis, Kline-Rogers, Mallya, Eagle, \& Erickson, (2005)

Again, it was found that women rated their symptoms as being less severe than men. Previous studies have also indicated that women perceiving pain and unexplained disorders often feel that their symptoms have been ignored by the physician and that they had difficulty being listened to seriously (Jerlock, Welin, Rosengren, \& Gaston-Johansson, 1998; Werner \& Malterud, 2003; Werner, Isaksen, \& Malterud, 2004). White and Johnson (2000) reported that men with chest pain, because of their self-conception of being healthy, sometimes deny pain and explain it in a more subtle way.

Given the patients' perceptions of illness and its symptoms as important determinants of behaviour which have been associated with a number of important outcomes, such as treatment adherence and functional recovery, there is the need to explore factors which influence patients' perceptions of illness symptoms with the view to promote treatment adherence and functional recovery. Specifically the study was designed to achieve the following objectives:

1. To find out the patient's perception of illness symptoms

2. To determine the prevalent nature of patients' cultural belief about illness

3. To examine the social support provisions available to patients in Oyo state hospitals.

4. To determine the individual and composite influence of Cultural beliefs, social support and societal ascribed roles on Patients' perception of illness symptom

Consequently the following research questions were posed:

1. What are the patients' perceptions of illness symptoms?

2. What is the prevalent nature of patients' cultural belief about illness?

3. What are the social support provisions available to patients in Oyo state hospitals.

4. What are the individual and composite influence of Cultural beliefs, social support and societal ascribed roles on Patients' perception of illness symptom

Also, the following hypotheses were designed:

1. There is no significant difference in male and female patients' perception of illness symptoms

2. Patients' age does not significantly influence their perception of illness symptoms.

\section{Procedure}

This study adopts descriptive survey research design. The target population for this study comprised all in-patients and out-patients in the forty-six State-owned hospitals, out of which nine hospitals were selected randomly. This resulted in the selection of the following hospitals:

i. Adeoyo State Hospital, Ring Road, Ibadan,

ii. Jericho General Hospital, Jericho, Ibadan.

iii. State and General Hospital Ogbomoso.

iv. State Hospital Oyo.

v. General Hospital, Iseyin.

vi. State Hospital, Saki.

vii. Sick Bay School of Nursing Eleyele, Ibadan.

viii. Jericho Nursing Home, Jericho Ibadan.

ix. Jericho Chest Hospital, Ibadan.

Also, fifty (50) in-patients and out-patients were selected by convenience sampling from each of the selected hospitals to participate in the study. Although, a total of 450 respondents were selected only the responses from 370 respondents were usable as some did not complete the questionnaire properly or refused to give necessary and relevant information. The demographic features of the eventual participants on this study are presented in table 1 .

The instrument for the study is a questionnaire titled "questionnaire on patients' perception of illness symptoms". It comprised five sections. Section A was designed to 
collect demographic information consisting items like sex, age, educational qualification and so on. Section B sought information on the respondent's perception of illness symptoms while section $\mathrm{C}$ sought information on societal scribed roles. Sections D and E were designed to obtain information on the social support available to the patient and his/her cultural beliefs about illness. The instrument used a 4-point Likert-type response format and were ascribed scores ranging from $4=$ strongly agree to $1=$ strongly disagree.

Table 1. Demographic summaries of the research participants

\begin{tabular}{|c|c|c|c|}
\hline Variables & Levels & Frequency & Percentage $\%$ \\
\hline \multirow{3}{*}{ Sex } & Male & 80 & 21.6 \\
\hline & Female & 290 & 78.4 \\
\hline & Total & 370 & 100.0 \\
\hline \multirow{5}{*}{ Age } & $18-30 y r s$ & 167 & 45.1 \\
\hline & $31-42 \mathrm{yrs}$ & 106 & 28.6 \\
\hline & $43-54 y r s$ & 39 & 10.5 \\
\hline & Above $55 \mathrm{yrs}$ & 58 & 15.7 \\
\hline & Total & 370 & 100.0 \\
\hline \multirow{6}{*}{$\begin{array}{c}\text { Educational } \\
\text { attainment }\end{array}$} & $\begin{array}{l}\text { No Formal } \\
\text { Education }\end{array}$ & 32 & 8.6 \\
\hline & Primary & 54 & 14.6 \\
\hline & Secondary & 110 & 29.7 \\
\hline & Polytechnic/NCE & 106 & 28.6 \\
\hline & University & 68 & 18.4 \\
\hline & Total & 370 & 100.0 \\
\hline \multirow{4}{*}{ Religion } & Christianity & 162 & 43.8 \\
\hline & Islam & 202 & 54.6 \\
\hline & Traditional & 6 & 1.6 \\
\hline & Total & 370 & 100.0 \\
\hline \multirow{7}{*}{ Occupation } & Artisanship & 113 & 30.5 \\
\hline & Civil Service & 96 & 25.9 \\
\hline & Petty Trading & 94 & 25.4 \\
\hline & Unemployed & 42 & 11.4 \\
\hline & Retiree & 8 & 2.2 \\
\hline & Others & 17 & 4.6 \\
\hline & Total & 370 & 100.0 \\
\hline \multirow{6}{*}{$\begin{array}{c}\text { Marital } \\
\text { status }\end{array}$} & Married & 289 & 78.1 \\
\hline & Single & 50 & 13.5 \\
\hline & Separated & 16 & 4.3 \\
\hline & Divorced & 3 & .8 \\
\hline & Widow & 12 & 3.2 \\
\hline & Total & 370 & 100.0 \\
\hline
\end{tabular}

These data are therefore displayed graphically below:

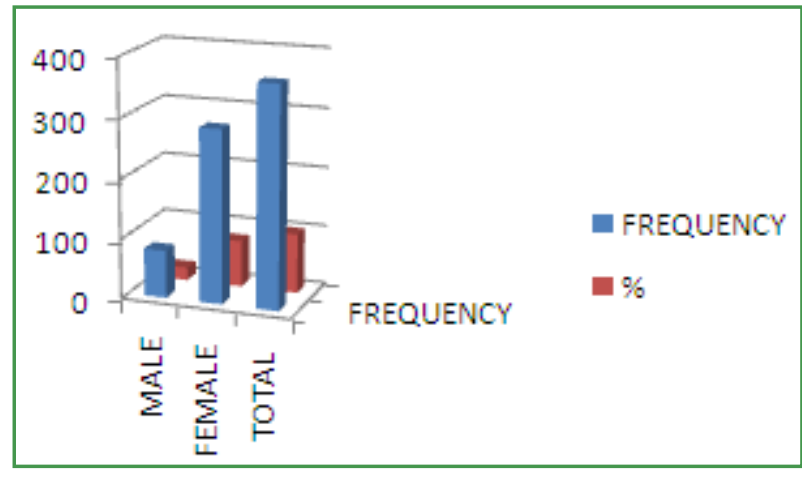

SEX

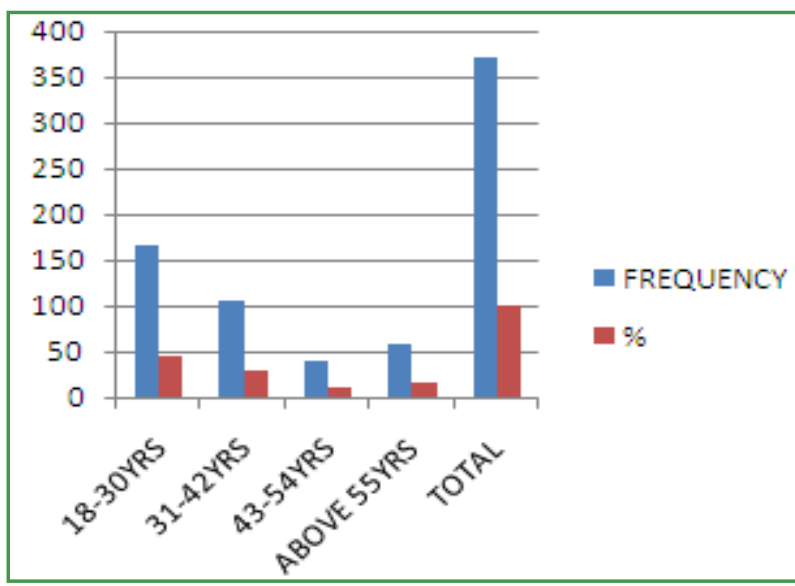

AGE

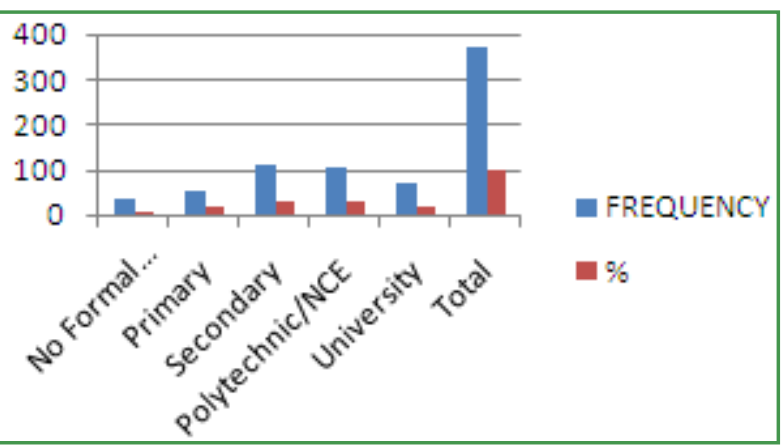

Educational Attainment

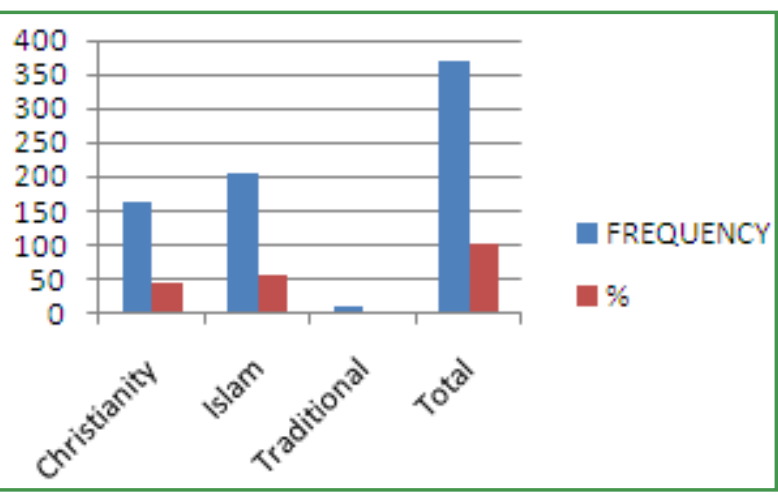

Religion 


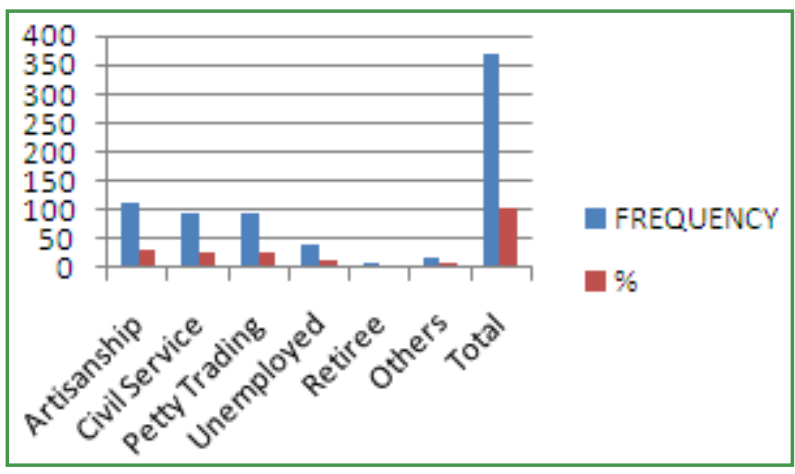

Occupation

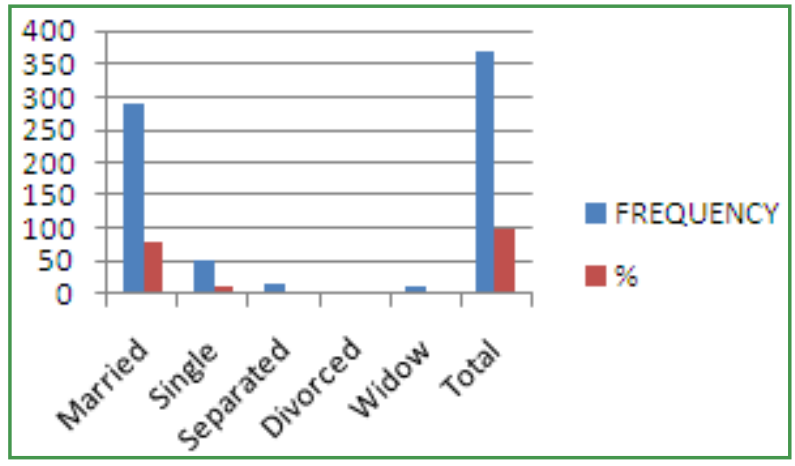

Marital

In order to ensure the validity of the instrument, the instrument was subject to validation exercise using expert judgment by given it to experts in Psychology and Tests and Measurement for expert scrutiny. All their contributions were noted and the corrections were effected to ensure content and construct validity. Further, a pilot test was carried out on the instrument using the test re-test method; the instrument was administered on patients not included among the selected hospitals to obtain the reliability coefficients ( $\mathrm{r}=0.71,0.79,0.76$ and 0.89$)$ for section $\mathrm{B}, \mathrm{C}$, $\mathrm{D}$ and $\mathrm{E}$ of the instrument respectively and these values were considered adequate to carry on with the study.

The researcher obtained a letter of introduction and to whom it may concern from the department which was submitted at the Ministry of Health and Oyo State Ethical Committee, for review and the researcher sought for their approval to administer the questionnaire in the selected hospitals, after which the researcher was given the permission to administer the questionnaire. Initially, the administration of the instruments was scheduled for three weeks, due to logistic reasons beyond the researcher control the administration of it went on for seven weeks. Four research-assistants were employed; they were tutored on what to do. The participants were duly informed about the purpose of the exercise. The consent form was attached to the questionnaire which was given to them and they were shown where to attest their signature, this was explained in the language they understood, and the content of the questionnaire was also translated into Yoruba language. Most essentially, the researcher encouraged the research assistants to establish good rapport with the participants in order to achieve the objective of the study.

The data collected were analysed thus; descriptive statistics of frequency distribution and percentage was used in analysing socio demographic information which was later presented in the form of tables. The use of t-test and chi-square analysis was adopted in testing the hypotheses while Multiple Regression Analysis and Analysis of Variance (ANOVA) were used in answering the four research questions. Hence, the distribution of all the variables in the study was examined at the significance level of 0.05 .

\section{Results}

\section{Research Question 1: What are the Patients' Perceptions of Illness Symptoms?}

To answer this research question, the patients' responses were scored in such a way that a "strongly agree" response was allotted 4, "agree", 3 and "Disagree", 2 while a "strongly disagree" response was allotted a score of 1 . These scoring procedures were transposed for the negative item, B4. The resulting scores were cumulated to constitute a measure of patients' perception of illness symptom. The respondents were categorized in such a way that those who scored 15 or less on the measure are said to possess negative perception of their illness symptom while those who scored 16 to 30 were said to be indifferent. Those who scored 31 and above on the measure were said to have positive perception. These categories were then given a descriptive analysis and the result is presented in table 2

Table 2. Patients' perception of illness symptoms

\begin{tabular}{|c|c|c|}
\hline & Frequency & Percent \\
\hline Negative & 11 & 2.8 \\
\hline Indifferent & 227 & 56.8 \\
\hline Positive & 162 & 40.5 \\
\hline Total & 400 & 100.0 \\
\hline
\end{tabular}

Table 2 shows the patients' perception of illness symptom. From the table it can be seen that most of the respondents $(56.8 \%)$ were indifferent to their illness symptom while $40.5 \%$ of the patients were actually positive. Only a meager $2.8 \%$ of the respondents seem to possess negative perception of their illness symptom.

\section{Research Question 2: What is the Prevalent Nature of Patients' Cultural Belief about Illness?}

To answer this research question, the patients' response to the 8-item section $\mathrm{C} 3$ of the questionnaire was given a descriptive analysis and the result is presented in table 3 . 
Table 3. Prevalent nature of patients' cultural belief about illness

\begin{tabular}{|c|c|c|c|c|c|c|c|c|c|c|c|}
\hline \multirow{2}{*}{$\mathrm{SN}$} & \multirow{2}{*}{ Patients' cultural belief about illness } & \multicolumn{2}{|c|}{ SA } & \multicolumn{2}{|c|}{ A } & \multicolumn{2}{|c|}{ D } & \multicolumn{2}{|c|}{ SD } & \multirow{2}{*}{ RSI } & \multirow{2}{*}{ 䒕 } \\
\hline & & f & $\%$ & $\mathrm{f}$ & $\%$ & $\mathrm{~F}$ & $\%$ & f & $\%$ & & \\
\hline 1 & $\begin{array}{l}\text { Pains are better not expressed in my society } \\
\text { even when it is critical }\end{array}$ & 122 & 30.5 & 83 & 20.8 & 70 & 17.5 & 108 & 27.0 & 0.643 & 2 \\
\hline 2 & $\begin{array}{l}\text { I see illness as spiritual and can only be cured } \\
\text { through spiritual means }\end{array}$ & 115 & 28.8 & 93 & 23.3 & 59 & 14.8 & 123 & 30.8 & 0.628 & 5 \\
\hline 3 & $\begin{array}{l}\text { I prefer the use of herbal and local concoction in } \\
\text { curing malaria, measles and other ailments }\end{array}$ & 107 & 26.8 & 78 & 19.5 & 94 & 23.5 & 108 & 27.0 & 0.619 & 6 \\
\hline 4 & Medicinal herbs are cheap and easy to procure & 86 & 21.5 & 140 & 35.0 & 61 & 15.3 & 99 & 24.8 & 0.638 & 3 \\
\hline 5 & $\begin{array}{l}\text { I only see a doctor if local herbs fail in curing } \\
\text { my ailment }\end{array}$ & 51 & 12.8 & 51 & 12.8 & 127 & 31.8 & 158 & 39.5 & 0.497 & 8 \\
\hline 6 & I use both local herbs and tablets concurrently & 146 & 36.5 & 122 & 30.5 & 48 & 12.0 & 64 & 16.0 & 0.730 & 1 \\
\hline 7 & $\begin{array}{l}\text { Certain diseases occur to serve as punishment } \\
\text { for breaching family taboos }\end{array}$ & 109 & 27.3 & 70 & 17.5 & 72 & 18.0 & 125 & 31.3 & 0.608 & 7 \\
\hline 8 & $\begin{array}{l}\text { Certain illnesses are caused by forces of evil } \\
\text { and cannot be cured through medical means } \\
\text { such as Abiku }\end{array}$ & 125 & 31.3 & 77 & 19.3 & 64 & 16.0 & 116 & 29.0 & 0.638 & 3 \\
\hline
\end{tabular}

Table 4. Social support provisions available to patients in Oyo state hospitals

\begin{tabular}{|c|c|c|c|c|c|c|c|c|c|c|}
\hline & Social support activities & \multicolumn{2}{|c|}{ SA } & \multicolumn{2}{|c|}{ A } & \multicolumn{2}{|c|}{$\mathrm{D}$} & \multicolumn{2}{|c|}{ SD } & \\
\hline & I got someone to: & $\mathrm{F}$ & $\%$ & $\mathrm{~F}$ & $\%$ & f & $\%$ & $\mathrm{~F}$ & $\%$ & \\
\hline 1 & Help me if confined to bed & 178 & 44.5 & 151 & 37.8 & 34 & 8.5 & 31 & 7.8 & 0.802 \\
\hline 2 & Take me to doctor & 174 & 43.5 & 149 & 37.3 & 39 & 9.8 & 32 & 8.0 & 0.795 \\
\hline 3 & Prepare my meals and help with daily chores & 184 & 46.0 & 143 & 35.8 & 44 & 11.0 & 24 & 6.0 & 0.808 \\
\hline 4 & Show love and affection to me & 219 & 54.8 & 149 & 37.3 & 11 & 2.8 & 12 & 3.0 & 0.868 \\
\hline 5 & Spend time with me even in my most miserable moments & 204 & 51.0 & 154 & 38.5 & 19 & 4.8 & 13 & 3.3 & 0.852 \\
\hline 6 & Listen to me, show concern for me and share my worries & 222 & 55.5 & 143 & 35.8 & 12 & 3.0 & 8 & 2.0 & 0.876 \\
\hline 7 & Confide ion or talk to about myself or my problems & 207 & 51.8 & 144 & 36.0 & 23 & 5.8 & 19 & 4.8 & 0.843 \\
\hline 8 & Understand my problems & 209 & 52.3 & 154 & 38.5 & 16 & 4.0 & 14 & 3.5 & 0.855 \\
\hline 9 & Give me advice I really want & 211 & 52.8 & 153 & 38.3 & 16 & 4.0 & 12 & 3.0 & 0.859 \\
\hline 10 & Give me information & 197 & 49.3 & 164 & 41.0 & 14 & 3.5 & 16 & 4.0 & 0.847 \\
\hline 11 & $\begin{array}{l}\text { My response to treatment is partly due to the love received from } \\
\text { my relations }\end{array}$ & 200 & 50.0 & 148 & 37.0 & 18 & 4.5 & 24 & 6.0 & 0.836 \\
\hline
\end{tabular}

Table 3 above shows the patients responses to their cultural beliefs toward illnesses. It can be seen from the table that the most prevalent cultural belief among the patients under study is "I sue both local herbs and tablets concurrently" with the highest RSI value of 0.730 and which was agreed to (strongly and ordinarily) by $66.0 \%$ of the respondents and only $28 \%$ of the respondents disagreed, others chose to abstain. Other prevalent beliefs include "Pains are better not expressed in my society even when it is critical", "Certain illnesses are caused by forces of evil and cannot be cured through medical means such as Abiku" and "Medicinal herbs are cheap and easy to procure" with RSI values of $0.643,0.638$ and 0.638 respectively. The least popular belief as expressed by the respondents is "I only see a doctor if local herbs fail in curing my ailment" with the least RSI value of 0.497 . This statement was agreed to (strongly and ordinarily) by $25.6 \%$ of the respondents.

\section{Research Question 3: What are the Social Support Provisions Available to Patients in Oyo State Hospitals?}

To answer this research question, the patients' response to section $\mathrm{C} 2$ of the questionnaire was given a descriptive analysis and the result is presented in table 4

From table 4 above, the patients indicate that the most prevalent form of social support available to the patients is "finding someone to listen to me, show concern for me and share my worries" closely followed by "finding someone to show love and affection to me". However, the least popular form according to the respondent is "finding some to take me 
to the doctor". Summarily the response were scored in such a way that 4 was allotted to SA, 3 to A, 2 to D and 1 to SD. The resulting scores were aggregated and were used to build a measure of social support available to the patients. Those who score 22 or below on the measure were said to receive low level of social support while those who score 23 or more on the measure were said to experience high level of social support. These two categories were then given a descriptive analysis and the result is presented in table 5 below

Table 5. Level of social support available to the patients

\begin{tabular}{|c|c|c|}
\hline & Frequency & Percent \\
\hline Low level & 20 & 5.0 \\
\hline High level & 380 & 95.0 \\
\hline Total & 400 & 100.0 \\
\hline
\end{tabular}

Table 5 shows the descriptive analysis of the level of social support available to the respondents. It can be seen from the table that most of the respondents $(95 \%)$ indicated that they experience high level of social support while only $5 \%$ claimed that they experience low level of social support.

\section{Research Question 4: What are the Individual and Composite Influence of Cultural Beliefs, Social Support and Societal Ascribed Roles on Patients' Perception of Illness Symptom?}

To answer this research question, a multiple stepwise regression analysis was conducted with patients' perception treated as the dependent variable while the patients' scores on cultural beliefs, social support and societal ascribed roles were inserted one after the other and the model evaluated at each stage. The resulting models are presented in table 6 .

Table 6 shows the models that resulted from the stepwise multiple regression used to determine the influence of Cultural beliefs, social support and societal ascribed roles on patients' perception of illness symptom. It can be seen from the table that out of the three factors, the societal ascribed roles seem to have the greatest influence on patients' perception of illness symptom with an Adjusted R-square value of 0.164 meaning that it can account for a minimum of $16.4 \%$ of any variance observed in the patients' perception of illness symptom. An addition of social support increased the observable variance to $17.3 \%$ meaning that it can only account for the marginal increase $(0.9 \%)$ in the observed variance. Also addition of cultural beliefs increased the observed variance to 19.2 meaning that Cultural beleiefs can account for $1.9 \%$ of the observed variance.

Table 6. Model Summary resulting from the stepwise Multiple regression

\begin{tabular}{|c|c|c|c|c|c|c|}
\hline \multirow{2}{*}{ Adjusted R Square } & \multirow{2}{*}{ Std. Error of the Estimate } & \multicolumn{4}{|c|}{ Change Statistics } \\
\cline { 3 - 7 } & & R Square Change & F Change & df1 & df2 & Sig. F Change \\
\hline .164 & 4.40793 & .166 & 79.407 & 1 & 398 & .000 \\
\hline .173 & 4.38382 & .011 & 5.389 & 1 & 397 & .021 \\
\hline .192 & 4.33363 & .021 & 10.249 & 1 & 396 & .001 \\
\hline
\end{tabular}

a. Predictors: (Constant), Societal ascribed roles

b. Predictors: (Constant), Societal ascribed roles, Social support available

c. Predictors: (Constant), Societal ascribed roles, Social support available, Cultural beliefs 


\section{Hypotheses}

\section{Hypothesis 1: There is no significant difference in male and female patients' perception of illness symptoms}

To test this hypothesis, the patients' scores on the perception of illness symptoms was subjected to a t-test of difference with their gender as the differentiating variable. The result is presented in table 7 .

Table 7. difference in male and female patients' perception of illness symptoms

\begin{tabular}{|c|c|c|c|c|c|c|}
\hline Sex & $\mathrm{N}$ & Mean & $\begin{array}{c}\text { Std. } \\
\text { Deviation }\end{array}$ & $\mathrm{T}$ & $\mathrm{Df}$ & $\mathrm{p}$ \\
\hline Male & 80 & 32.8625 & 4.34798 & 1.801 & 365 & 0.098 \\
\hline
\end{tabular}

Table 7 presents the result of a test of difference in male and female perception of illness symptom. From the table it can be seen that although the mean score of the male patients is slightly higher that those of the male students, this difference is not significant as $\mathrm{t}_{(365)}=1.801, \mathrm{p}>.05$.

\section{Hypothesis 2: Patients' age does not significantly influence their perception of illness symptoms.}

To test this hypothesis, a test of difference in the patients' perception of illness symptoms on the basis of their age group was carried out via Analysis of Variance (ANOVA). The result is presented in table 8 .

Table 8. Influence of patients' age on perception of illness symptoms

\begin{tabular}{|c|c|c|c|c|c|}
\hline & $\begin{array}{c}\text { Sum of } \\
\text { Squares }\end{array}$ & Df & $\begin{array}{c}\text { Mean } \\
\text { Square }\end{array}$ & F & Sig. \\
\hline $\begin{array}{c}\text { Between } \\
\text { Groups }\end{array}$ & 246.248 & 3 & 82.083 & 3.661 & .013 \\
\hline Within Groups & 7937.196 & 354 & 22.421 & & \\
\hline Total & 8183.444 & 357 & & & \\
\hline
\end{tabular}

Table 8 shows the result of the test of influence of age on patients' perception of illness symptoms. It can be seen from the table that the F-value obtained is 3.661 at $\mathrm{p}$-value $=0.013$. Sincde the P-value is lower than 0.05 , it can be concluded that age has a significant influence on patients' perception of illness symptoms. To locate the groups that a significantly different from each othert a multiple comparison test was conducted via Tukey HSD. The result is presented in table 9

Table 9. Multiple comparison test of the influence of age on patients' perception of illness symptoms

\begin{tabular}{|c|c|c|c|c|c|c|}
\hline \multirow{2}{*}{ (I) Age } & \multirow{2}{*}{ (J) Age } & \multirow{2}{*}{ Mean Difference (I-J) } & \multirow{2}{*}{ Std. Error } & \multirow{2}{*}{ Sig. } & \multicolumn{2}{|c|}{ 95\% Confidence Interval } \\
\hline & & & & & Lower Bound & Upper Bound \\
\hline \multirow{3}{*}{$18-30 y r s$} & $31-42 \mathrm{yrs}$ & 1.35294 & .59437 & .106 & -.1813 & 2.8872 \\
\hline & $43-54 \mathrm{yrs}$ & $2.39247^{*}$ & .92560 & .050 & .0032 & 4.7817 \\
\hline & above $55 \mathrm{yrs}$ & 1.51754 & .72582 & .158 & -.3560 & 3.3911 \\
\hline \multirow{3}{*}{$31-42 \mathrm{yrs}$} & $18-30 y r s$ & -1.35294 & .59437 & .106 & -2.8872 & .1813 \\
\hline & $43-54 \mathrm{yrs}$ & 1.03953 & .97113 & .708 & -1.4672 & 3.5463 \\
\hline & above $55 \mathrm{yrs}$ & .16460 & .78306 & .997 & -1.8567 & 2.1859 \\
\hline \multirow{3}{*}{$43-54 \mathrm{yrs}$} & $18-30 \mathrm{yrs}$ & $-2.39247^{*}$ & .92560 & .050 & -4.7817 & -.0032 \\
\hline & $31-42 \mathrm{yrs}$ & -1.03953 & .97113 & .708 & -3.5463 & 1.4672 \\
\hline & above $55 \mathrm{yrs}$ & -.87493 & 1.05671 & .841 & -3.6026 & 1.8527 \\
\hline \multirow{3}{*}{ above $55 \mathrm{yrs}$} & $18-30 y r s$ & -1.51754 & .72582 & .158 & -3.3911 & .3560 \\
\hline & $31-42 \mathrm{yrs}$ & -.16460 & .78306 & .997 & -2.1859 & 1.8567 \\
\hline & $43-54 \mathrm{yrs}$ & .87493 & 1.05671 & .841 & -1.8527 & 3.6026 \\
\hline
\end{tabular}

*. The mean difference is significant at the 0.05 level. 


\section{Multiple Comparisons}

The table 9 above shows the multiple comparisons of the differences in the perception of illness symptom between different age groups of the patients. It can be seen from the table that only the It appears that the older one gets, the more positive he/she is in terms of his/her perception of illness symptom. However, only the difference between those of 18 to $30 \mathrm{yrs}$ and those of 43 to 54Yrs are significant

\section{Discussion}

Research has extended the description of Illness beyond diseases to include "how the sick person, and the members of the family or wider social network perceive, live with, and respond to symptoms and disability (Kleinman, 1988). He argued that the illness experience includes, categorizing, and explaining the forms of distress caused by those path physiological processes. It therefore follows that any productive effort to improve the well-being of patients must include an understanding of their perception of illness symptom.

Consequently, research question 1 was posed to appraise the patients' perception of illness symptom in the hospitals under study. The result shows that a considerable percentage of the patients were positive in their perception of their illnesses and that only a meager proportion possessed negative perception. This is not uncommon in African culture, where people commonly belief that their perception of illnesses affect their recovery rates, in fact, research confirms that all cultures have systems of health beliefs to explain what causes illness, how it can be cured or treated, and who should be involved in the process(McLaughlin \& Braun, 1998). Also, Vaughn, Jacquez and Baker (2009) posited that this may have major implications for medical professionals because over time, attributions have been found to play an essential role in the formation of beliefs concerning health and illness which in turn becomes reciprocal and health beliefs form a cognitive schema that influences the way that people make attributions. Through test of hypotheses, male and female patients' perceptions of illness symptoms were significantly not different but in terms of age, it was found that the older patients seem to have more positive perceptions.

Research question 2 was used to survey the prevalent cultural beliefs the patients have about illness. The result showed that the most prevalent cultural belief among the patients is that they use both local herbs and tablets concurrently. Other prevalent beliefs include the fact that pains were better not expressed in the society even when it is critical and that certain illnesses are caused by forces of evil and cannot be cured through medical means such as Abiku. These results are in agreement with findings of Chipfakacha (1994) that attribution believes of Africans with regard to illnesses include the fact that illnesses are due to magic and evil spirits or causes which relate to conflict and tension between good/evil and harmony/disharmony. In order to effectively treat these illnesses, therefore, remedies must be both material (e.g. herbal remedy) and spiritual (e.g., amulets) explanations and techniques (Vaughn, Jacquez \& Baker, 2009).

Further, social support has been found to having significant influence on subjective well-being (Kahn, Hessling, \& Russell, 2003). Other research findings also hold that social support seems to exert influence on health both directly and indirectly through certain cognitive mechanisms, coping strategies, and health behaviours (Cohen \& Wills, 1985; Davis \& Swan, 1999). Consequently, research question 3 was posed to appraise the social support provisions available to patients in Oyo state hospitals. The results show that most of the patients indicated that they enjoy high level of social support. This may account for the positive perception they have on their illness symptoms. However another research question was posed to find out which of patients' cultural beliefs, social support and societal ascribed roles affect the patients' perception of their illness symptom most. The result showed that their societal ascribed roles seem to be the one which affects their perception of illness most.

It can be concluded that whatever the patients' cultural belief may be, social support given to the social support provisions and the societal ascribed roles will determine the way the patient perceive his/her illness. This will in turn affect the rate of recovery and response to treatment. It can be recommended that social workers need to focus attention on patients' perception of illness symptom in order to aid recovery and response to treatment.

\section{REFERENCES}

Broadbent, E., Petrie K. J., Main, J. and Weinmann J. (2006). The brief illness perception questionnaire. Journal of Psychosomatic Research, 60, 631- 637 .

Chipfakacha V. (1994) The role of culture in primary health care. South African Medical Journal 84(12): 860-1.

Cohen, S. \& Wills, T.A. (1985). Stress, Social Support and the Buffering Hypothesis. Psychological Bulletin, 98, 310-357.

Davis, M.S. \& Swan, P.D. (1999). Association of Negative and Positive Social Ties with Fibrinogen Levels in Young Women. Health Psychology, 18,131-139

DiMatteo, M. R. \& Martin, L. R. (2002). Health Psychology. Boston: Allyn and Bacon

Giri, P., Poole, J., Nightingale P. and Robertson, A. (2009). Perceptions of illness and their impact on illness absence. Occup Med (Lond) (2009)59 (8): 550-555. doi:10.1093/occmed/kqp123

Jennings D. (1986). The confusion between disease and illness in clinical medicine. Canadian Medical Association Journal, 135, 865-870.

Johnson, R. (2002). The concept of illness behavior: a brief 
chronological account of four key discoveries. Veterinary Immunology and Immunopathology, 87 (3-4): 443 450.doi:10.1016/ S0165-2427(02)00069-7.

Kahn, J. H., Hessling, R. M., \& Russell, D. W. (2003). Social support, health, and well being among the elderly: What is the role of negative affectivity?. Personality and Individual Differences 35 , $5-17$.

Kelley, K. W., Bluthe, R. M. , Dantzer, R., Zhou, J. H., Shen, W. H., Johnson, R. W., Broussard, S. R. (2003). Cytokine-induced illness behavior. Brain Behaviour Immunology, 17 (Suppl. 1): 112-118. doi:10.1016/S0889-1591(02)00077-6.

Kleinman, A. (1988). The illness narratives: suffering, healing, and the human condition. New York: Basic Books
Mc Laughlin, L., \& Braun, K. (1998). "Asian and Pacific Islander cultural values: Considerations for health care decision-making." Health and Social Work, 23 (2), 116-126.

Petrie K. J. and Weinmann, J. (2006) Why illness perceptions matter. Clinical Medicine, 6, 536-539.

Petrie, K. J. and Weinman, J. (2012). Patients' Perceptions of Their Illness: The Dynamo of Volition in Health Care. Current Directions in Psychological Science, 21(1), 60-65

Vaughn,L. M., Jacquez, F. and Baker, R. C. (2009). Cultural Health Attributions, Beliefs, and Practices: Effects on Healthcare and Medical Education. The Open Medical Education Journal, 2009, 2, 64-74 